\title{
AVALIAÇÃO DA FORMAÇÃO INTERPROFISSIONAL NO ENSINO SUPERIOR EM SAÚDE: ASPECTOS DA COLABORAÇÃO E DO TRABALHO EM EQUIPE
}

\author{
Rinaldo Henrique Aguilar-DA-Silva* \\ LuCIana TeIXeIra SCAPIN** \\ Nildo Alves Batista***
}

Recebido: jun. 2010

Aprovado: ago. 2010

\begin{abstract}
* Mestre e Doutor em Genética e Evolução (UFSCar), Pós-doutorando em Ensino em Ciências da Saúde pela Universidade Federal de São Paulo (UNIFESP), Especialista em Administração Universitária e Gestão Acadêmica, Professor da Faculdade de Medicina de Marília (Famema). E-mail: aguilar@famema.br

** Mestre em Ciências da Saúde - Área de Concentração Saúde Mental - Especialista em Psicopedagogia - Especialista em Assistência ao Psicótico - Professora da Faculdade de Ciências Médicas e da Saúde de Juiz de Fora (FCMS/JF). E-mail:

*** Mestre em Medicina (Pediatria) pela Universidade de São Paulo (USP), Doutor em Medicina (Pediatria) pela USP, Livre-Docente em Educação Médica pela Universidade Federal de São Paulo, Professor Titular da Universidade Federal de São Paulo (UNIFESP) - Departamento de Saúde, Educação e Sociedade, Pró-Reitor Adjunto de Graduação, Coordenador do Programa de Pós-Graduação, nível Mestrado, em Ensino em Ciências da Saúde e Diretor Acadêmico do Campus Baixada Santista da UNIFESP. E-mail: nbatista@unifesp.br
\end{abstract}

Resumo: Acredita-se que a educação interprofissional pode reforçar as atitudes para o trabalho em equipe e colaboração, levando a uma melhor assistência ao paciente após a formatura. Em outras palavras, o objetivo fundamental da educação interprofissional é a formação de estudantes de graduação na área da saúde mais preparados para a prática interprofissional. Este estudo procurou determinar quais as atitudes dos estudantes em relação à educação interprofissional para aspectos de colaboração e trabalho em equipe na Faculdade de Ciências Médicas e da Saúde de Juiz de Fora (FCMS / JF). Os dados foram coletados utilizando-se uma escala de percepção do trabalho interprofissional (RIPLS) e realização de grupo focal. Os resultados desta pesquisa indicam que os estudantes de graduação em ciências da saúde estão abertos para a aprendizagem compartilhada.

Palavras-chave: Educação Interprofissional. Avaliação. Educação Superior.

\section{EVALUATION OF INTERPROFESSIONAL EDUCATION IN UNDERGRADUATE HEALTH SCIENCE: ASPECTS OF COLLABORATION AND TEAMWORK}

Abstract: There is a belief that interprofessional education will enhance attitudes toward teamwork and collaboration, leading to improved patient care upon graduation. In other words, the key objective of interprofessional education is to create undergraduate health science students who are prepared for interprofessional practice. This study sought to establish what the attitudes of undergraduates toward interprofessional education for aspects of collaboration and teamwork in the Faculdade de Ciências Médicas e da Saúde de Juiz Fora (FCMS/JF). Data were collected using the Readiness for Interprofessional Learning Scale (RIPLS) questionnaire survey and focus group interviews. The findings of this research indicate that undergraduate health science students are open to the notion of shared learning.

Key words: Interprofessional education. Evaluation. University education. 


\section{INTRODUÇÃO}

O Ensino Interprofissional em Saúde pode ser conceituado como uma proposta onde duas ou mais profissões aprendem juntas sobre o trabalho conjunto e sobre as especificidades de cada uma, na melhoria da qualidade no cuidado ao paciente (UNIFESP, 2009). É considerada um estilo de educação que prioriza o trabalho em equipe, a integração e a flexibilidade da força de trabalho que deve ser alcançada com um amplo reconhecimento e respeito às especificidades de cada profissão.

Em vários países, experiências de ensino interprofissional na graduação em saúde (HIND et al, 2003; MCNAIR et al, 2005; COOPER et al, 2005; GOELEN et al, 2006) apresentaram mudanças consistentes no perfil dos profissionais formados. Estas possibilitam a discussão sobre a intencionalidade deste tipo de formação, apontando o desafio de se ter domínio de conhecimentos, habilidades e atitudes para atuar com competência, com qualidade formal e política e, ao mesmo tempo, responder às necessidades sociais. Abordam ainda como as questões ético-políticas, podem auxiliar na otimização dos recursos de saúde e desenvolver a consciência nos atos/escolhas praticados já que passam a ser discutidos criticamente em equipe.

A formação interprofissional em saúde faz parte do currículo da Faculdade de Ciências Médicas e da Saúde de Juiz de Fora (FCMS/JF) através da realização do Programa Integrador, junto a Atenção Primária à Saúde, que tem como objetivo contribuir para a formação integrada de profissionais de saúde e para a melhoria do Sistema de Saúde do município de Juiz de Fora.

Este é um dos componentes centrais da estrutura curricular que engloba os cursos de Medicina, Enfermagem, Fisioterapia, Farmácia e Odontologia visando à indissociabilidade entre teoria-prática, a integração da Faculdade ao meio social local e regional, bem como a integração entre os cursos.

Busca-se com esse Programa proporcionar condições de inserção em contextos reais de aprendizagem, por meio de ações em diferentes comunidades, integração aos serviços de saúde, aprendizado das ações preventivas e de promoção da saúde, e atuação em equipes interprofissionais constituídas por estudantes das diferentes áreas desde o início da formação.

Nessa perspectiva, torna-se importante o desenvolvimento da capacidade de trabalho em equipe, observação do meio sociocultural, identificação das características da população local, bem como de suas demandas. O Programa acontece nos seis semestres iniciais dos cursos e tem como núcleos: a) integração entre ensino, pesquisa/práticas investigativas e extensão; b) estreita arti- 
culação entre teoria e prática; c) o desenvolvimento das atividades por equipes interprofissionais sob supervisão docente e com a orientação da equipe de saúde dos núcleos de Saúde da Família; c) participação, em nível crescente de complexidade, do atendimento ambulatorial de nível primário e secundário, em atividades de promoção de saúde da criança, do adolescente, do adulto, da mulher e do idoso.

As peculiaridades do setor saúde tornam especialmente interessante o estudo da formação interprofissional, uma vez que apresenta grande especialização em categorias e procedimentos e ao mesmo tempo vem sendo pressionado para diminuir a fragmentação de suas abordagens aos pacientes, seja por razões de eficácia ou mesmo de equacionamento de custos. Trata-se ainda de um setor no qual prevalecem profissionais liberais de várias formações que obedecem e, frequentemente, privilegiam orientações exteriores à organização, como aquelas provenientes dos conselhos de classe. Além disso, os membros das equipes de saúde atuam sobre problemas de extrema complexidade, realizando tarefas interdependentes e sobre as quais possuem controle relativo e cujos desdobramentos são sempre incertos, já que condicionados por vários fatores ( $\mathrm{D}^{\prime} \mathrm{AMOUR}, 1997$ ).

Alguns estudos têm demonstrado que dentre as diferentes categorias profissionais predomina a tendência em satisfazer suas aspirações e manter sua autonomia, em detrimento da colaboração profissional (D'AMOUR, 1997). Tal postura espelha a própria constituição das profissões que possuem em comum os interesses pragmáticos de garantia de mercado de trabalho. Conforme nos lembram estudos realizados pela sociologia das profissões, o profissionalismo constitui-se na história como estratégia de retenção do conhecimento, buscando torná-lo o mais específico e misterioso possível, permanecendo acessível a poucos e assim garantindo reserva de mercado (MACHADO, 1995).

Conforme discutido por Almeida Filho (1997), a interdisciplinaridade ou a formação interprofisisonal não se efetiva através de princípios ou de intenções genéricas desenvolvidas em textos de pesquisadores bem intencionados. A troca efetiva e colaboração entre disciplinas e profissões serão possíveis somente pela ação de agentes concretos que irão ou não consubstanciar práticas mais ou menos integradas. Como ilustrado pelo autor, é somente através da concretude dos aparelhos cognitivos de indivíduos que transitem em diferentes áreas que ocorrerão os diferentes graus de interação e colaboração entre disciplinas e profissões.

Segundo Carpenter (1995), a convivência e a educação interprofissional, modificam atitudes e percepções negativas entre os profissionais de saúde re- 
mediando falhas na confiança e comunicação. Freeth (2002) também aponta que esta estratégia propicia maior conscientização e preparo para a prática, contribuindo para a socialização entre os profissionais de saúde. Cooper et al (2005), avaliando a educação interprofissional com estudantes de medicina, enfermagem, fisioterapia e terapia ocupacional na universidade de Liverpool, constatou o desenvolvimento de maior confiança e reforço da identidade profissional.

Assim, a inserção responsável e comprometida de estudantes e docentes nos cenários reais, desde a primeira série dos cursos na FCMS/JF visa a integração teórico/prática, ensino/serviço e educação interprofissional sendo o alicerce em torno do qual tece-se uma nova forma de ser, fazer, conhecer e conviver. Segundo Gyamarti (1986), a educação interprofissional possibilita integrar cuidado especializado com cuidado holístico, opondo-se ao reducionismo e a fragmentação da visão especializada.

Para que isso ocorra, é necessário mais do que reunir estudantes de cursos diferentes em atividades conjuntas. Devem ser desenvolvidos recursos cognitivos, afetivos e psicomotores dos estudantes, tendo em mente que a identificação e o atendimento das necessidades de saúde das pessoas em questão, nas situações apresentadas, respeita o grau de autonomia e domínio de cada profissão. Sendo assim, torna-se possível entender como as múltiplas dimensões de qualquer problema de saúde e a sua abordagem por distintas profissões se apresentam e se articulam na determinação do processo saúde-doença.

\section{OBJETIVO}

Avaliar as percepções dos estudantes dos diferentes cursos da saúde da FCMS/JF em relação ao trabalho em equipe e a efetiva colaboração entre eles durante o desenvolvimento das atividades propostas pelo Programa Integrador.

\section{MÉTODO}

\subsection{Natureza do estudo}

Tratou-se de uma análise de pesquisa avaliativa, o que segundo Contandriopoulos et al. (1997) significa "examinar através de um procedimento científico as relações que existem entre os diferentes componentes de uma intervenção" e para tanto foram utilizadas as abordagens de pesquisa quantitativa e qualitativa que pretende se aproximar de uma realidade com a finalidade de conhecê-la (MINAYO; SANCHES, 1993). 


\subsection{Campo do estudo e aspectos éticos}

O campo é o recorte espacial que corresponde à abrangência, em termos empíricos, ao objeto de estudo; este compreende o contexto onde se desenvolve o objeto, sendo o espaço onde serão obtidos os dados empíricos sobre o mesmo (MINAYO, 2005).

Assim, o campo determinado para este estudo foi onde se desenvolveram as atividades do Programa Integrador dos cursos de Medicina, Enfermagem, Fisioterapia, Farmácia e Odontologia da FCMS/JF.

Esta pesquisa foi aprovada pelo comitê de ética da Universidade Federal de São Paulo (UNIFESP) para o Projeto "Estudo de Avaliação da Formação Interprofissional na Graduação em Saúde: contribuição para delimitação do objeto" sob o número - 1879/09. Os que concordaram participar do estudo assinaram Termo de Consentimento Livre Esclarecido.

\subsection{Coleta dos dados}

Os dados quantitativos foram coletados junto aos estudantes egressos do Programa Integrador $(\mathrm{n}=135)$ utilizando-se a Readiness Interprofessional Learning Scale (RIPLS) (PARSELL; BLIGH, 1999) com nove questões estruturadas abordando os aspectos do trabalho em equipe e efetiva colaboração com outros profissionais de saúde (Anexo A).

Os dados qualitativos foram obtidos através da realização de grupo focal com a presença de dois estudantes de cada um dos cursos que participaram da pesquisa $(n=10)$. A pergunta utilizada como disparador foi: o que você entende por colaboração interprofissional e trabalho em equipe?

\subsection{Análise dos dados}

A pesquisa quantitativa se apoia nos pressupostos do positivismo, é objetivista, dedutiva, mede opiniões e dados procurando descobrir e classificar a relação entre as variáveis (relação de causa e efeito), empregando recursos técnicos de estatística. As nove questões respondidas pelos estudantes foram estruturadas em cinco pontos da escala de Likert. As respostas foram compiladas em uma planilha Excel e analisadas utilizando qui-quadrado para testar as diferenças entre grupos. As frequências das respostas também foram avaliadas. Ao utilizar este teste analisamos os dados para verificar a significância estatística e determinar o grau de confiança para aceitar ou rejeitar a hipótese. 
O método utilizado para a análise qualitativa baseou-se na análise de conteúdo, modalidade temática, com ênfase na abordagem qualitativa de pesquisa social (GOMES, 2005). Esta técnica consiste em descobrir os "núcleos de sentido" que compõem a comunicação e cuja presença ou frequência de aparição pode significar alguma coisa para o objetivo analítico escolhido.

\section{RESULTADOS}

\subsection{Dados quantitativos}

As respostas obtidas revelaram que no que se refere à eficácia do trabalho em grupo a maioria dos estudantes concordaram ou concordaram fortemente que a aprendizagem com outros estudantes irão ajudá-los a tornarem-se melhores no trabalho em equipe ( $\mathrm{Q} 1 \mathrm{n}=122,90,37 \%$ ) e que o desenvolvimento de competências profissionais são essenciais para que todos possam aprender sobre o cuidado ao paciente $(\mathrm{Q} 3 \mathrm{n}=119,88,14 \%)$. Foram positivas as opiniões sobre os benefícios do trabalho em equipe e a maioria dos estudantes concordou ou concordou fortemente que a aprendizagem compartilhada os ajuda a compreender as próprias limitações $(\mathrm{Q} 4 \mathrm{n}=88,65,18 \%)$ porém $(16,29 \%)$ se posicionou como neutra nesta questão. A maioria também indicou que os pacientes se beneficiam quando o trabalho é realizado em conjunto na resolução dos problemas (Q5 $\mathrm{n}=101,74,81 \%)$. No que se refere ao aumento da capacidade na resolução de problemas clínicos quando se trabalha em equipe foi observado que houve a concordância da maioria (Q6 n=76, 56,29\%). Entretanto, observou-se que $43 \%$ mantiveram-se neutros ou em desacordo com este item. A maioria dos estudantes também concordou ou concordou fortemente que o aprendizado de habilidades e atitudes deve ser desenvolvido em conjunto com estudantes de outros cursos (Q8 n=97, 71,85\%). Já no que se refere ao relacionamento com outros profissionais de saúde, a maioria dos estudantes concordou ou concordou fortemente que, para trabalhar em pequeno grupo, são necessários a confiança e o respeito mútuo (Q2 n = 113, 83,70\%). E que a aprendizagem com outros estudantes da área da saúde, antes da formatura, irá melhorar os relacionamentos durante a vida profissional $(\mathrm{Q} 7 \mathrm{n}=$ $105,77,77 \%$ ). Além disto, a maioria concordou que a aprendizagem compartilhada irá ajudá-los a pensar positivamente sobre os outros profissionais (Q9 $\mathrm{n}=81,60 \%)$; porém, $21(15,55 \%)$ dos estudantes se manifestaram neutros e $33(24,45 \%)$ não concordaram com este ponto. 


\subsection{Dados qualitativos}

Foi realizada a transcrição da gravação do grupo focal e, após isto, a leitura do material buscando uma compreensão global, na qual se privilegiou não apenas o conteúdo, como também sua lógica. Em seguida foram identificados núcleos de sentido ou eixos que estruturavam os depoimentos, em torno dos quais se agrupavam características comuns (GOMES, 2005) da temática colaboração e trabalho em grupo.

Todos apontaram em suas falas a importância do trabalho em equipe e a colaboração entre diferentes profissões: "Hoje em dia não é mais possível trabalhar sozinho, em todas as profissões principalmente as ligadas à saúde trabalhar com outros profissionais é fundamental para o sucesso profissional". Ressaltaram também as dificuldades encontradas durante este processo: "Não é fácil trabalhar em equipe, principalmente quando a equipe é formada por estudantes de vários cursos. É uma guerra de egos e de poder". Os estudantes do curso de Medicina e Odontologia discordaram dos demais quanto à necessidade da inserção deste tipo de formação já nas séries iniciais dos cursos: "Não acho importante aprender já no início do curso este tipo de colaboração, acredito que nos anos finais do curso, quando eu já estiver no estágio poderei aprender mais sobre o trabalho em grupo". "Na minha profissão o trabalho em equipe é bastante restrito e durante o curso às vezes vejo que este tipo de atividade acaba tomando muito tempo que poderia ser utilizado para aprender outras coisas". Parece não existir clareza por parte de todos os estudantes de quais são as tarefas específicas de cada profissional para o estabelecimento das competências profissionais : "Durante o trabalho proposto, muitas vezes não sei qual é a minha função e me sinto inibido em ultrapassar o meu papel e ser criticado por isso". Entretanto foi fortemente apontado por todos que o trabalho em equipe tem proporcionado um aprendizado efetivo: "Tenho aprendido muito com os colegas de outros cursos. Nunca pensei que pudesse ser melhor aprender assim".

\section{DISCUSSÃO}

De modo geral os resultados obtidos neste estudo revelaram a positividade da formação interprofissional em saúde no que se refere à colaboração e ao trabalho em equipe. Entretanto, para se estabelecer um trabalho coeso e eficiente é necessário o envolvimento de todos, busca pela satisfação no trabalho, o treinamento e desenvolvimento de competências, a criação de si- 
nergia entre os participantes, a definição de papéis, além do acompanhamento e avaliação de resultados.

A complexidade encontrada na formação de recursos humanos para a área da saúde pareceu minimizar-se nas respostas das questões e nas falas dos estudantes quando questionados sobre as possibilidades do trabalho em equipe. Assim, o preconceito existente em formar juntos profissionais que irão trabalhar juntos acabou por desfazer-se neste processo avaliativo.

Casto e Júlia (1994) também avaliando educação interprofissional e a prática colaborativa, apontaram que os profissionais desenvolvem a capacidade de lidar com problemas que excedem a capacidade de uma profissão isoladamente. Isto fortalece o compartilhamento de competências colaborativas porque inverte a lógica da formação tradicional em saúde possibilitando que as profissões aprendam juntas sobre o trabalho conjunto e sobre as especificidades de cada uma na melhoria da qualidade do cuidado ao paciente (BARR, 1998).

Portanto, estudantes de diferentes cursos ao levarem em conta as necessidades de saúde individuais e coletivas para propor medidas que ampliem o cuidado e, consequentemente, que melhorem a qualidade de vida das pessoas, aprendem a aprender juntos sobre si, sobre o outro e sobre as diferentes profissões. Isto também foi verificado por McNair et al (2005) que observaram a ampliação das experiências no trabalho de equipe na prática interprofissional com estudantes de medicina, farmácia e fisioterapia.

As relações de importância culturalmente imputadas a algumas profissões, como a medicina e odontologia, ainda aparecem refletidas na resistência dos estudantes quanto à necessidade do início destas atividades.

Interessantemente, houve uma distribuição equitativa no que se referiu ao entendimento de que a aprendizagem compartilhada aumenta a vontade e capacidade para compreender os cuidados de saúde e os problemas clínicos dos pacientes. Isto se contrapõe aos achados de Horsburgh et al (2001) e Reid et al (2006) que relataram que estudantes de medicina discordam desta afirmativa por entenderem que o médico ocupa papel central na coordenação da equipe e resolução dos problemas do paciente.

Como então estabelecer espaços de negociação para uma atuação integrada? Para que haja uma equipe de integração e não equipe agrupamento (PEDUZZI, 1998), há necessidade de uma construção dos sujeitos de diferentes cursos, considerando que todas as profissões têm sua relevância. Essa nova construção requer articulação das ações e a interação dos agentes envolvidos no processo, sendo esta última a mais difícil, pois não está "normatizada" a 
priori e requer um compromisso ético e respeito com o outro, com cada um e com todos da equipe e acima de tudo com os pacientes, entendendo que a ação de todos resultará na maior eficiência do atendimento e resolução das necessidades de saúde dos pacientes segundo preconizam as diretrizes do Sistema Único de Saúde (SUS).

Uma questão se coloca em relação à consolidação destas práticas de saúde que compõem um modelo calcado nos princípios e diretrizes do SUS. É a problemática da força de trabalho, sendo que esta deve ser enfrentada tanto do ponto de vista das práticas como da formação de pessoal. Aponta-se ao longo dos anos de implementação do SUS que a formação da força de trabalho em saúde proporcionada pelo aparelho formador não é adequada às necessidades de saúde da população brasileira nem às necessidades dos serviços (NOGUEIRA, 2002).

Assim, as universidades formam, separados, profissionais que necessitarão trabalhar juntos. Nessa perspectiva, há necessidade de se redirecionar a formação inicial, colocando em questão os modelos e valores a serem construídos. Para atender a esta reorganização, pautando-se pela ética nos processos de intervenção, é necessário construir e re-construir os processos de trabalho e competências profissionais, integrando a formação à realidade e dos serviços da comunidade com a presença de todos os profissionais necessários na equipe.

O principal aspecto positivo da atuação em equipe é a possibilidade de colaboração de várias especialidades que denotam conhecimentos e qualificações distintas. Esse mesmo aspecto, entretanto, pode dificultar a compreensão mútua e a possibilidade de uma tarefa uniforme, pelas diferenças próprias de cada área, tanto em nível do conhecimento em si, como da própria tarefa. Isto pode ser verificado nos discursos apresentados, revelando que existe pouca clareza sobre as atividades que devem ser realizadas e que isto gera insegurança. Assim, esclarecer o nível de complexidade e, sobretudo, de autonomia dos estudantes frente às tarefas propostas pode garantir maior conforto e sucesso do Programa.

A competência de cada profissional, isoladamente, não dá conta da complexidade do atendimento das necessidades de saúde, portanto é necessário flexibilidade nos limites das competências para proporcionar uma ação integral.

O trabalho multiprofissional refere-se à recomposição de diferentes processos de trabalho que, concomitantemente devem flexibilizar a divisão do trabalho; preservar as diferenças técnicas entre os trabalhadores especializa- 
dos; argüir a desigualdade na valoração dos distintos trabalhos e respectivos agentes, bem como nos processos decisórios e tornarem consideração a interdependência dos trabalhos especializados no exercício da autonomia técnica, dada a necessidade de autonomia profissional para a qualidade da intervenção em saúde (PEDUZZI, 2000, p. 6).

A compreensão e a definição clara dos papéis profissionais associados a determinada tarefa são indispensáveis nas instituições de saúde. Principalmente porque a indefinição ou a ambiguidade relativa ao papel profissional pode gerar conflitos na equipe ao acumular-se expectativas inadequadas ou mal delimitadas entre seus membros. Ressalta-se que a delimitação do papel profissional acompanha as expectativas dos outros membros da equipe quanto ao papel que o profissional em questão deve exercer, acrescidas das próprias expectativas do profissional sobre sua capacidade de realização e de interpretação das expectativas dos outros (CAMPOS, 1997).

Esta falta de compreensão está fortemente ligada ao entendimento do conceito de competência profissional. Segundo Tsuji e Aguilar-da-Silva (2010) competência profissional é a capacidade circunstancial de mobilizar articuladamente recursos cognitivos, afetivos e psicomotores para a resolução de uma situação-problema. Assim, entende-se que os profissionais não são competentes em $100 \%$ das abordagens e isto parece não ser confortável para a maioria destes. Considerar que nesta era da gestão do conhecimento, onde a quantidade de informação cresce a cada segundo, o sucesso pessoal/profissional está também vinculado ao trabalho do outro é um grande desafio.

A identificação da importância dessa abordagem passa também pela própria definição de equipe que abrange uma ampla gama semântica - interdisciplinar, multidisciplinar, intradisciplinar, transdisciplinar, intraprofissional e interprofissional, embora possam ser apontadas algumas tentativas de distinção entre si. De uma forma geral, equipe tem sido definida como um grupo de profissionais que atuam de forma independente em um mesmo ambiente de trabalho, utilizando-se de comunicações informais.

Baptista (2002) assume que a identidade profissional é construída na interação do eu com a sociedade. Sua ênfase recai naquilo que as pessoas têm em comum, enquanto grupo sociocultural, e as diferencia em relação àqueles que atuam em outros ramos de atividade.

Assim, ao distinguir o grupo de trabalho, do trabalho de colaboração em equipe, é possível verificar que o grupo de trabalho, na maioria das vezes, refere-se à união de profissionais de saúde para a realização de tarefas que ocorrerem em um mesmo ambiente. Isto necessariamente não significa parti- 
lha de suas tarefas, constatações e responsabilidades que visariam aprimorar o serviço.

Robbins (2004) afirma que as equipes são capazes de melhorar o desempenho dos indivíduos quando a tarefa requer múltiplas habilidades, julgamentos e experiência, pelo fato de serem mais flexíveis reagindo melhor às mudanças.

Para o trabalho interprofissional a equipe passa a ter uma outra importância. É necessário existir a construção de uma visão compartilhada. Segundo Senge (1998):

Uma Visão Compartilhada não é uma idéia. Nem que essa idéia seja tão importante quanto a liberdade. Pelo contrário, é uma força no coração das pessoas, uma força de impressionante poder. Pode ser inspirada por uma idéia, mas quando evolui - quando é estimulante o suficiente para obter o apoio de mais de uma pessoa - deixa de ser uma abstração. Torna-se palpável. As pessoas começam a vê-la como se existisse. Poucas forças, se é que existe alguma, nas questões humanas, são tão poderosas quanto uma visão compartilhada.

Parece não haver consenso de quando se deve iniciar a formação interprofissional em saúde e alguns autores (HORSBURGH et al, 2001; PARSELL; BLIGH, 1999; PIRRIE et al, 1998) defendem a ideia de que é melhor que seja introduzida durante o estágio ou na pós-graduação. Entretanto, os resultados encontrados indicaram que os estudantes estão abertos à possibilidade deste tipo de aprendizagem e que as crenças e valores podem sem trabalhados ao longo do curso propiciando a formação de profissionais mais competentes e preocupados com as necessidades do sistema de saúde e da qualidade do atendimento de seus pacientes.

Neste estudo, a formação interprofissional no Programa Integrador está localizada dentro da Estratégia de Saúde da Família vinculada á atenção primária à saúde.

Segundo Schraiber et al (1996) o cuidado primário em saúde, por parecer tratar-se de um serviço simples, usando geralmente poucos equipamentos, tem sido visto como assistência simplificada. No entanto, apresenta complexidade na articulação de diversos saberes e múltiplos profissionais, o que irá se refletir na atuação do profissional que precisa, a partir dessa nova concepção, desenvolver uma adequada abordagem e efetiva transformação nesse nível de atendimento, uma sofisticada síntese de saberes e complexa interação de ações: individuais e coletivas; curativas e preventivas; assistenciais e educativas buscando o desenvolvimento do trabalho em equipe. 
Segundo Ferreira et al (2007) a diversificação de cenários de prática traz consigo um aspecto delicado, elemento essencial da parceria, que consiste na necessidade de articulação entre a academia e o serviço de saúde, estratégia tão importante quanto difícil de implantar, porque os profissionais de cada uma dessas áreas obedecem a direções distintas, cumprem funções diferentes, são remunerados para ações específicas o que por vezes pode acabar por dificultar a formação das equipes.

Para minimizar este aspecto, faz-se essencial o estabelecimento de políticas que busquem a efetivação das equipes de saúde. Segundo Guizardi (2006), as estratégias de governo no campo das políticas públicas com ênfase nas interfaces entre saúde, formação e prática profissional estão para além dos programas de formação ou das políticas de reestruturação de grades curriculares. Estas estratégias envolvem domínios de saber, vinculações com regras morais e certos tipos de conhecimento que definem o campo de possibilidade de emergência das políticas públicas na saúde. Assim, estas políticas públicas configuram-se como rede discursiva e moral, que conformam ações e produzem sujeitos. Portanto, estamos abordando um processo de sujeição que institui certo lugar de estudante, de docente, profissionais da saúde e de pesquisador.

É nesta relação de complementaridade e interdependência e ao mesmo tempo de autonomia relativa com um saber próprio que contatamos através desta pesquisa que o trabalho de equipe é indispensável para o sucesso dos profissionais da saúde. Articular estes distintos aspectos não é um empreendimento rápido e requer esforço contínuo para que em todos os espaços possíveis possamos construir a ideia de equipe integração. Integrar conhecimentos disponíveis nos espaços de trabalho, nos espaços de formação, nos espaços de produção de conhecimento, especialmente nos espaços de construção de cidadania.

\section{CONSIDERAÇÕES FINAIS}

Esta avaliação do Programa Integrador evidenciou que essa estratégia tem como essência o trabalho em equipe, a união dos integrantes, na busca de um objetivo comum, caracterizando a formação Interprofissional em Saúde. Além disso, indicou ser necessário haver flexibilidade para possibilitar a articulação com os serviços de Atenção Primária à Saúde. Demonstrou que o trabalho em equipe potencializa a possibilidade do alcance das competências profissionais, o estabelecimento de vínculo e a criação de laços de compromisso 
com co-responsabilização entre profissionais e estudantes que são elementos essenciais para o alcance dos objetivos da Formação Interprofissional em Saúde. Isto só é possível como uma mudança de atitude das Instituições de Ensino Superior na perspectiva da Formação Interprofissional permeada pela pactuação com os serviços de saúde. O profissional de saúde e os estudantes devem atuar na ampliação dos referenciais com que trabalham e estudam e reconhecer os limites da sua ação individual e isolada para atender a todo o universo de necessidades do usuário, contribuindo, assim, para a atenção integral. Os estudantes revelaram que, para a melhoria do trabalho em equipe, é preciso privilegiar relações amistosas, na perspectiva de articulações de ações permeadas pela prática da comunicação intra e inter equipe. Nessa direção, o trabalho em equipe é norteado pelo investimento na criatividade, na busca de novos mecanismos para o aprimoramento da qualidade da produção de cuidado em saúde e da Formação Interprofissional.

\section{REFERÊNCIAS}

ALMEIDA FILHO, N. Transdisciplinaridade e saúde coletiva. Ciência \& Saúde Coletiva, Rio de Janeiro, v. 2, n. 1/2, p. 5-20, 1997.

BARR, H. Competent to collaborate; towards a competency-based model for interprofessional education. Journal of Interprofessional Care, London, UK, v. 12, n. 2, p. 181-188, 1998.

BAPTISTA, M.T.D.S. O estudo de identidades individuais e coletivas na constituição da história da Psicologia. Memorandum, Belo Horizonte, v. 2, p. 31-8, 2002. Disponível em: <http://www.fafich.ufmg.br/ memorandum/ artigos02/baptista01.htm>. Acesso em: 9 set. 2008.

CAMPOS, G. W. S. Subjetividade e administração de pessoal: considerações sobre modos de gerenciar o trabalho em equipes de saúde. In: MERHY, E. E.; ONOCKO, R. (Orgs). Agir em saúde: um desafio para o público. São Paulo: Hucitec, 1997. p. 71-112.

CARPENTER, J. Interprofessional education for medical and nursing students: evaluation of a programme. Medical Education, Oxford, GB, n. 29, p. 265-272, 1995. 
CASTO, R. M.; JULIA, M. C. Interprofessional care and collaborative practice. Pacific Grove: Brooks/Cole Publishing Company, 1994.

CONTANDRIOPOULOS, A. P. et al. A avaliação na área da saúde: conceitos e métodos. In: HARTZ, Z. M. A. (Org.). Avaliação em saúde: dos modelos conceituais a prática na análise da implantação de programas. Rio de Janeiro: Edit. FIOCruz, 1997. p. 29-47.

COOPER, H.; SPENCER-DAWE, E.; MCLEAN, E. Beginning the process of teamwork: design, implementation and evaluation of an inter-professional education intervention for first year undergraduate students. Journal of Interprofessional Care, London, UK, v. 19, n. 5, p. 492-508, 2005.

D'AMOUR, D. Structuration de la collaboration interprofessionelle dans les services de santé de première ligne au Québec. 1997. Tese (Doutorado) -Université de Montreal, Montreal/Canadá, 1997.

FREETH, D. et al. A critical review of interprofessional education, 2002. Disponível em: <www.caipe.org.uk/publications>. Acesso em: 05 jul. 2006.

FERREIRA, R.C.; SILVA R.F.; AGUERA, C.B. Formação do Profissional Médico: A Aprendizagem na Atenção Básica de Saúde. Revista Brasileira de Educação Médica, Rio de Janeiro, v. 31, n. 1, p.52-59, 2007.

GOELEN, G. et al. Measuring the effect of interprofessional problem-based learning on the attitudes of undergraduate health care students. Medical Education, Oxford, GB, v. 40, v. 6, p. 555-61, 2006.

GOMES, R. et al. Organização, processamento, análise e interpretação dos dados: o desafio da triangulação. In: MINAYO, M. C. S. et al (Org.). Avaliação por triangulação de métodos: abordagem de programas sociais. Rio de Janeiro: Fio Cruz, 2005. p. 185 - 221.

GUIZARDI, F. L. et al. A formação de profissionais orientada para a integralidade e as relações político institucionais na saúde: uma discussão sobre a interação ensino-trabalho. In: CECCIM, R, B.; PINHEIRO, R; MATTOS, R. A. (Org.). Ensinar saúde: a integralidade e o SUS nos cursos de graduação na área da saúde. Rio de Janeiro: IMS/Uerj, 2006.

GYAMARTI, G. The teaching of the professions: an interdisciplinary approach. Higher Education Review, Oxford, GB, v. 18, n. 2, p. 33-43, 1986. 
HIND, M. et al. Interprofessional perceptions of health care students. Journal of Interprofessional Care. London, UK, v.17, n. 1, p. 21-34,Feb. 2003.

HORSBURGH, M.; LAMDIN, R.; WILLIAMSON, E. 'Multiprofessional learning: the attitudes of medical, nursing and pharmacy students to shared learning', Medical Education, Oxford, GB, n. 35, p. 876-883, 2001.

MACHADO, M. H. Sociologia das profissões: uma contribuição ao debate teórico. In: MACHADO, M. H. (Org.). Profissões de saúde: uma abordagem sociológica. Rio de Janeiro: Fiocruz, 1995.

MCNAIR, R.; STONE, N.; SIMS, J.; CURTIS, C. Australian evidence for interprofessional education contributing to effective teamwork preparation and interest in rural practice. Journal of Interprofessional Care, London, UK, n. 19 , p. 579-94, 2005.

MINAYO, M. C. S. et al. Métodos, técnicas e relações em triangulação. In: MINAYO, M. C. et al. (Org.) Avaliação por triangulação de métodos: abordagem de programas sociais. Rio de Janeiro: Fiocruz, 2005.

MINAYO M. C. S., SANCHES, O. Qualitativo-quantitativo: oposição ou complementaridade? Cadernos de Saúde Pública, Rio de Janeiro, v. 9, n. 3, p. 239-248, jul./set. 1993.

NOGUEIRA, R. P. (Coord.). Avaliação das tendências e prioridades sobre recursos humanos de saúde. Brasília: Organização Pan-Americana da Saúde, 2002.

PARSELL, G.; BLIGH, J. The development of a questionnaire to assess the readiness of health care students for interprofessional learning (RIPLS). Medical Education, Oxford, GB, n. 33, p. 95-100, 1999.

PEDUZZI, M. Equipe multiprofissional de saúde: a interface entre trabalho e interação. Campinas, 1998. 254p. Tese (Doutorado) - Faculdade de Ciências Médicas, UNICAMP, 1998.

PEDUZZI, M. A inserção do enfermeiro na equipe de saúde da família, na perspectiva da promoção da saúde. In: SÃO PAULO. Secretaria do Estado da Saúde. Seminário: o enfermeiro no Programa de Saúde da Família. São Paulo, 2000. 
PIRRIE, A.; WILSON, V.; HARDEN, R.; ELSEGOOD, J. Promoting cohesive practice in health care. Medical Teacher, London, UK, v. 20, n. 5, p. 405-416, 1998.

REID, R.; BRUCE, D.; ALLSTAFF, K.; MCLERNON, D. Validating the Readiness for Interprofessional Learning Scale (RIPLS) in the postgraduate context: Are health professionals ready for IPL? Medical Education, Oxford, GB, v. 40, n. 5, p. 415-422, 2006.

ROBBINS, S. Fundamentos do comportamento organizacional. 7. ed. São Paulo: Pearson Education, 2004.

SCHRAIBER, L. B.; NEMES, M. I. B.; GONÇALVES, R. B. M. Saúde do adulto: programas e ações na unidade básica. Saúde em Debate, série didática “3”. São Paulo: Hucitec, 1996.

SENGE, P.M. A quinta disciplina: arte, teoria e prática da organização da aprendizagem. São Paulo: Best Seller, 1998.

TSUJI, H.; AGUILAR-DA-SILVA, R. H. Aprender e ensinar na escola vestida de branco: do modelo biomédico ao humanístico. São Paulo: Phorte, 2010 .

UNIFESP. O projeto pedagógico do Campus Baixada Santista. Disponível em: $<$ http://www.baixadasantista.unifesp.br/projpedag.php $>$. Acesso em: 06 jul. 2009. 


\section{ANEXO A - READINESS INTERPROFESSIONAL LEARNING SCALE (RIPLS) (PARSELL; BLIGH, 1999) PARA ASPECTOS DA COLABORAÇÃO E DO TRABALHO EM EQUIPE}

Q1 - O aprendizado junto com outros estudantes irá me ajudar a tornar-me um membro efetivo de uma equipe de saúde

\begin{tabular}{|l|l|l|l|l|}
\hline $\begin{array}{l}\text { Concordo } \\
\text { Fortemente }\end{array}$ & Concordo & Neutro & Discordo & $\begin{array}{l}\text { Discordo } \\
\text { Fortemente }\end{array}$ \\
\hline
\end{tabular}

Q2 - Para a aprendizagem em pequenos grupos de trabalho os estudantes precisam da confiança e do respeito uns dos outros

\begin{tabular}{|l|l|l|l|l|}
\hline $\begin{array}{l}\text { Concordo } \\
\text { Fortemente }\end{array}$ & Concordo & Neutro & Discordo & $\begin{array}{l}\text { Discordo } \\
\text { Fortemente }\end{array}$ \\
\hline
\end{tabular}

Q3 - Em um trabalho de equipe as competências são essenciais para que todos os alunos possam aprender sobre os cuidados de saúde

\begin{tabular}{|l|l|l|l|l|}
\hline $\begin{array}{l}\text { Concordo } \\
\text { Fortemente }\end{array}$ & Concordo & Neutro & Discordo & $\begin{array}{l}\text { Discordo } \\
\text { Fortemente }\end{array}$ \\
\hline
\end{tabular}

Q4 - A aprendizagem compartilhada vai me ajudar a entender as minhas próprias limitações

\begin{tabular}{|l|l|l|l|l|}
\hline $\begin{array}{l}\text { Concordo } \\
\text { Fortemente }\end{array}$ & Concordo & Neutro & Discordo & $\begin{array}{l}\text { Discordo } \\
\text { Fortemente }\end{array}$ \\
\hline
\end{tabular}

Q5 - Os pacientes acabam tendo benefícios para a sua saúde quando os estudantes trabaIham em conjunto para resolver os problemas

\begin{tabular}{|l|l|l|l|l|}
\hline $\begin{array}{l}\text { Concordo } \\
\text { Fortemente }\end{array}$ & Concordo & Neutro & Discordo & $\begin{array}{l}\text { Discordo } \\
\text { Fortemente }\end{array}$ \\
\hline
\end{tabular}

Q6 - A aprendizagem compartilhada com outros estudantes da área de saúde aumenta a minha vontade e capacidade para compreender os cuidados de saúde e os problemas clínicos

\begin{tabular}{|l|l|l|l|l|}
\hline $\begin{array}{l}\text { Concordo } \\
\text { Fortemente }\end{array}$ & Concordo & Neutro & Discordo & $\begin{array}{l}\text { Discordo } \\
\text { Fortemente }\end{array}$ \\
\hline
\end{tabular}

Q7 - A aprendizagem com outros estudantes da área da saúde antes da formatura irá meIhorar os relacionamentos durante a vida profissional

\begin{tabular}{|l|l|l|l|l|}
\hline $\begin{array}{l}\text { Concordo } \\
\text { Fortemente }\end{array}$ & Concordo & Neutro & Discordo & $\begin{array}{l}\text { Discordo } \\
\text { Fortemente }\end{array}$ \\
\hline
\end{tabular}


Q8 - Comunicação e habilidades devem ser aprendidas junto com outros estudantes da área da saúde

\begin{tabular}{|l|l|l|l|l|}
\hline $\begin{array}{l}\text { Concordo } \\
\text { Fortemente }\end{array}$ & Concordo & Neutro & Discordo & $\begin{array}{l}\text { Discordo } \\
\text { Fortemente }\end{array}$ \\
\hline
\end{tabular}

Q9 - A aprendizagem compartilhada irá me ajudar a pensar positivamente sobre os outros profissionais

\begin{tabular}{|l|l|l|l|l|}
\hline $\begin{array}{l}\text { Concordo } \\
\text { Fortemente }\end{array}$ & Concordo & Neutro & Discordo & $\begin{array}{l}\text { Discordo } \\
\text { Fortemente }\end{array}$ \\
\hline
\end{tabular}

